\title{
In leukemia, knock-down of the death inducer-obliterator gene would inhibit the proliferation of endothelial cells by inhibiting the expression of CDK6 and CCND1
}

\author{
Honghua Cao ${ }^{\text {Equal first author, } 1}$, Lilan Wang ${ }^{\text {Equal first author, } 1}{ }^{1}$, Chengkui Geng ${ }^{2}$, Man Yang ${ }^{3}$, Wenwen Mao ${ }^{4}$, Linlin Yang ${ }^{5}$, Yin \\ $\mathrm{Ma}^{1}{ }^{1}$, Ming $\mathrm{He}^{1}$, Yeying Zhou ${ }^{1}$, Lianqing Liu ${ }^{1}$, Xuejiao $\mathrm{Hu}^{1}{ }^{1}$, Jingxing Yu ${ }^{6}$, Xiufen Shen ${ }^{7}$, Xuezhong Gu ${ }^{8}$, Liefen \\ Yin ${ }^{\text {Corresp., } 6}$, Zhenlei Shen ${ }^{\text {Corresp. } 1}$ \\ ${ }^{1}$ Department of Hematology, The third Affiliated Hospital of Kunming Medical University, Kunming, China \\ 2 Department of Orthopedics, Yan'an Hospital of Kunming City, the Affiliated Hospital of Kunming Medical University, Kunming, China \\ 3 Department of Endocrinology, the Affiliated Hospital of Yunnan University \& the Second People's Hospital of Yunnan Province, Kunming Yunnan \\ 650021], Kunming, Yunnan, China \\ 4 Department of Geriatics, The second Hospital of Kunming, Kunming, China \\ 5 Department of Gynecology, The third Affiliated Hospital of Kunming Medical University, Kunming, China \\ 6 Department of Hematology, The second Affiliated Hospital of Kunming Medical University, Kunming, China \\ 7 Department of laboratory, The second Affiliated Hospital of Kunming Medical University, Kunming, China \\ 8 Department of Hematology, The First people hospital in Yunnan province, Kunming, China \\ Corresponding Authors: Liefen Yin, Zhenlei Shen \\ Email address: ylfynkm@126.com, szl1020@163.com
}

Background: Endothelial cells (ECs) are a critical component of the hematopoietic niche, and the crosstalk between ECs and leukemia was reported recently. This study aimed to determine the genes involved in the proliferation inhibition of endothelial cells in leukemia.

Methods: Human umbilical vein endothelial cells (HUVEC) were cultured alone or cocultured with K562 cell lines. The GeneChip assays were performed to identify the differentially expressed genes. The Celigo, MTT assay and flow cytometric analysis were used to determine the effect of RNAi DIDO on cell growth and apoptosis. The differentlyexpressed genes were verified by qRT-PCR (quantitative real-time PCR) and western-blot.

Results: In K562-HUVEC co-cultured cell lines, 323 down-regulated probes were identified and the extracellular signal-regulated kinase 5 (ERK5) signaling pathway was significantly inhibited. Among the down-regulated genes, the death inducer-obliterator gene $(D I D O)$ is a part of the centrosome protein and may be involved in cell mitosis. As shown in the public data, leukemia patients with lower expression of DIDO showed a better overall survival (OS). The HUVEC cells were infected with shDIDO lentivirus, reduced expression, inhibited proliferation, and increased apoptosis was observed in shDIDO cells. In addition, the expression of Cyclin-Dependent Kinase 6 (CDK6) and Cyclin D1 (CCND1) genes was inhibited in shDIDO cells. At last, the public ChIP-seq data were used to analyze the regulators that bind with DIDO, the H3K4me3 and PollI (RNA polymerase II) signals were found near the Exon1 and exon2 sites of DIDO.

Conclusion: The knock-down of DIDO will inhibit the proliferation of endothelial cells in the leukemia environment. The expression of DIDO may be regulated by H3K4me3 and the inhibition of DIDO may lead to the down-regulation of CDK6 and CCND1. However, how DIDO interacts with CDK6 and CCND1 needs further study.

Peer) reviewing PDF | (2021:02:58541:3:0:NEW 18 Dec 2021) 
2 In leukemia, knock-down of the death inducer-

3 obliterator gene would inhibit the proliferation of

4 endothelial cells by inhibiting the expression of CDK6

5 and CCND1

6

7

8

Honghua $\mathrm{Cao}^{1 *}$, Lilan Wang ${ }^{*}$, Chengkui Geng ${ }^{2 *}$, Man Yang ${ }^{3}$, Wenwen $\mathrm{Mao}^{4}$, Linlin Yang ${ }^{5}$, Yin $\mathrm{Ma}^{1}$, Ming $\mathrm{He}^{1}$, Yeying Zhou ${ }^{1}$, Lianqing $\mathrm{Liu}^{1}$, Xuejiao $\mathrm{Hu}^{1}$, JingxingY $\mathrm{u}^{6}$, Xiufen Shen ${ }^{7}$, Xuezhong $\mathrm{Gu}^{8}$, Liefen Yin $^{6 \#}$, Zhenglei Shen ${ }^{1 \#}$

1. Department of Hematology, the third Affiliated Hospital of Kunming Medical University, Kunming 650118, China;

2. Department of Orthopedics, Yan'an Hospital of Kunming City, the Affiliated Hospital of Kunming Medical University, Kunming 650051, China;

3. Department of Endocrinology, the Affiliated Hospital of Yunnan University \& the Second People's Hospital of Yunnan Province, Kunming Yunnan 650021;

4. Department of Geriatics, The second Hospital of Kunming, Kunming 650024, China;

5. Department of Gynecology, the third Affiliated Hospital of Kunming Medical University, Kunming 650118, China;

6. Department of Hematology, The second Affiliated Hospital of Kunming Medical University, Kunming 650031, China;

7. Department of laboratory, the second Affiliated Hospital of Kunming Medical University, Kunming 650031, China;

8. Department of Hematology, the First people hospital in Yunnan province, Kunming, 650032, China.

* Honghua Cao, Lilan Wang and Chengkui Geng contributed equally to this work

\# Corresponding authors:

1. Zhenglei Shen, Kunzhou Road, Kunming, Yunnan, 650118, China; Email: szl1020@163.com

2. Liefen Yin, Dianmian Street, Kunming, Yunnan, 650101, China 
35

36

37

67

\section{Abstract}

Background: Endothelial cells (ECs) are a critical component of the hematopoietic niche, and the cross-talk between ECs and leukemia was reported recently. This study aimed to determine the genes involved in the proliferation inhibition of endothelial cells in leukemia.

Methods: Human umbilical vein endothelial cells (HUVEC) were cultured alone or cocultured with K562 cell lines. The GeneChip assays were performed to identify the differentially expressed genes. The Celigo, MTT assay and flow cytometric analysis were used to determine the effect of RNAi DIDO on cell growth and apoptosis. The differently expressed genes were verified by qRT-PCR (quantitative real-time PCR) and western-blot.

Results: In K562-HUVEC co-cultured cell lines, 323 down-regulated probes were identified and the extracellular signal-regulated kinase 5 (ERK5) signaling pathway was significantly inhibited. Among the down-regulated genes, the death inducer-obliterator gene (DIDO) is a part of the centrosome protein and may be involved in cell mitosis. As shown in the public data, leukemia patients with lower expression of DIDO showed a better overall survival (OS). The HUVEC cells were infected with shDIDO lentivirus, reduced expression, inhibited proliferation, and increased apoptosis was observed in shDIDO cells. In addition, the expression of CyclinDependent Kinase 6 (CDK6) and Cyclin D1 (CCND1) genes was inhibited in shDIDO cells. At last, the public ChIP-seq data were used to analyze the regulators that bind with DIDO, the H3K4me3 and PolII (RNA polymerase II) signals were found near the Exon1 and exon2 sites of $D I D O$.

Conclusion: The knock-down of DIDO will inhibit the proliferation of endothelial cells in the leukemia environment. The expression of DIDO may be regulated by $\mathrm{H} 3 \mathrm{~K} 4 \mathrm{me} 3$ and the inhibition of $D I D O$ may lead to the down-regulation of $C D K 6$ and $C C N D 1$. However, how $D I D O$ interacts with $C D K 6$ and $C C N D 1$ needs further study.

\section{Introduction}

The increased number of circulating endothelial cells (ECs) in the peripheral blood was detected in multiple myeloma ${ }^{[1]}$, myelodysplasia ${ }^{[2]}$, and acute myeloid leukemia patients ${ }^{[3]}$. Besides, the higher level of circulating ECs and endothelial precursor cells (EPCs) was associated with more aggressive disease and shorter survival ${ }^{[4]}$ in chronic lymphocytic leukemia.

The cross-talk between leukemia cells and endothelial cells was reported recently. The endothelial cells (ECs) provide a fertile niche that will promote the proliferation of primitive and aggressive leukemia cells ${ }^{[5]}$. Besides, ECs could elaborate on angiocrine factors, which will take part in the reconstitution of normal and malignant stem/progenitor cells ${ }^{[6]}$. ECs provide critical support for the survival and progression of leukemia stem cells (LSCs) ${ }^{[7]}$, which would promote regeneration of leukemia ${ }^{[8,9]}$. On the other hand, the leukemic blasts could secrete numerous 
74 cytokines, which will augment the proliferation of microvascular endothelial cells in primary

75

76

77

78

79

80

81

82

83

84

85

86

87

88

89

90

91

92

93

94

95

96

97

98

99

100

101

102

103

104

105

106

107

108

109

110

111

112

113

acute myelocytic leukemia (AML) cells ${ }^{[10]}$. Leukemia may induce the activation of resting ECs and these activated ECs would protect the leukemia cells from chemotherapy injury ${ }^{[11]}$. Due to the protective effect of ECs in leukemia microenvironment ${ }^{[12]}$, vascular targeted drugs may be a new strategy for AML treatment decisions.

Leukemia-derived ECs may originate from bone marrow-derived hemangioma blast progenitor cells ${ }^{[13]}$, as the BCR-ABL fusion transcript in ECs was found derived from bone marrow progenitor cells ${ }^{[5]}$. Besides, the AML cells could integrate into vasculature and fuse with ECs in vivo, and the AML cells could differentiate into endothelial-like cells in vitro ${ }^{[14]}$.

A better understanding of the interaction between ECs and leukemia may inspire the design of innovative therapies for leukemia. Niche target treatment may help restore damaged vascular microenvironment, increase chemotherapy delivery and increase treatment responses. To determine the targets which will inhibit the ECs may be a new direction. In this study, we tried to investigate the genes involved in the interaction between the ECs and leukemia cells by GeneChip. The proliferation of HUVEC cells was inhibited when co-cultured with the K562 cells. A death inducer obliterator gene (DIDO) showed lower transcript abundance in HUVECK562 co-cultured cells. Although we have not found a report about the mechanism of DIDO in leukemia, there were reports showing that DIDO was involved in the development of solid tumors, such as bladder cancer ${ }^{[15]}, \mathrm{RCC}{ }^{[16]}$, and Melanoma ${ }^{[17]}$. In this study, we investigated the role of $D I D O$ in endothelial cells in the leukemia environment.

\section{Materials \& Methods}

\section{Cell lines and cell culture}

Human umbilical vein endothelial cells (HUVEC) and Human myeloid leukemia cell line (K562) were purchased from the American Type Culture Collection (Rockville, MD). The cell lines were maintained using RPMI (Roswell Park Memorial Institute) 1640 medium (Gibco Co, USA) supplemented with $10 \% \mathrm{FBS}$ at $37{ }^{\circ} \mathrm{C}$ in a humidified atmosphere containing $5 \% \mathrm{CO}_{2}$. The HUVEC and K562 cell lines were mixed and co-cultured for 4 days. CCK-8 Cell Counting Kit-8 (CCK-8) was used to determine the cell viability by colorimetric assays at $450 \mathrm{~nm}{ }^{[18]}$.

For RNA extraction and analysis of cell proliferation and apoptosis, the suspension of K562 cells was removed. Then the HUVEC cells in the two groups (HUVEC vs. HUVEC-K562) were washed by PBS buffer and collected for further experiments. The HUVEC cells were digested with pancreatin before RNA extraction.

\section{Plasmid constructs and transfection}

For gene knockdown, the GV115 vector was used in this study, which used the green fluorescent protein as a reporter gene, and the multiple cloning sites were driven by a human U6 promoter.

The $D I D O$ was targeted by the shRNA sequences of 5'- GGATGAGACTCATTCAGAA- 3'. The sequence was cloned into the multiple cloning sites by restriction enzyme of AgeI and EcoRI.

Peer] reviewing PDF | (2021:02:58541:3:0:NEW 18 Dec 2021) 
114 Plasmid transfection was performed as a former study ${ }^{[19]}$. The cell lines were seeded into 96-

115 well plates. After transfection for $2 \sim 3 \mathrm{~d}$, the GFP was observed under a fluorescence microscope.

116 The cells were used for further studies when the cell density in the wells reached 70-90\%.

117 Celigo and MTT assays

118 Cells were inoculated into the 96-well plates (2000 cells / well) and three repeats were taken.

119 The cell numbers were measured by Celigo Imaging Cytometer ${ }^{[19]}$ and the numbers were

120 recorded for 5 days. The cell numbers were normalized to the cell numbers on the first day after

121 seeding.

122 MTT (3-(4,5-dimethylthiazol-2-yl)-2,5-diphenyl tetrazolium bromide) assay ${ }^{[20]}$ was performed

123 to analyze the proliferation of cells. $1 \mathrm{mg} / \mathrm{mL}$ MTT was added to each well and incubated at

$12437^{\circ} \mathrm{C}$ for 4 hours. Then the culture medium was removed and the DMSO (150 $\mu$ l) was added into

125 each well, and then the plate was shaken for 3 minutes. The Tecan Infinite M2009PR plate

126 reader was used to measure the absorbance at $490 \mathrm{~nm} / 570 \mathrm{~nm}$.

127 Cell apoptosis analysis

128 The cell apoptosis was measured following the manufacturer's instructions of Annexin-FITC

129 Apoptosis Detection Kit (BD Biosciences, Franklin Lake, NJ, U.S.A.). Cells were cultured in a

130 96-well plate for 3-5 days in the $37{ }^{\circ} \mathrm{C}$ incubator, and then the cells were harvested and washed

131 in PBS. Cells were added to $0.5 \mathrm{ml}$ binding buffer and Annexin V-FITC, then the cells were

132 stained in the dark for $15 \mathrm{~min}$ at room temperature. Cells stained by Annexin V-FITC were

133 considered apoptotic cells ${ }^{[21]}$ which were measured by a BD Accuri ${ }^{\text {TM }}$ C6 flow cytometer (BD

134 Biosciences).

135 To analysis the cell apoptosis, Caspase 3/7 enzyme activity was measured by Caspase-Glo® 3/7

136 Assay (Promega, G8091). Caspase-Glo 3/7 reagent was added to the sample with a volume ratio

137 of $1: 1$, and the cells were incubated for another $1 \mathrm{~h}$ at $37{ }^{\circ} \mathrm{C}$. The Tecan Infinite M2009PR plate

138 reader was used to detect the luminescence in each well at $490 \mathrm{~nm} / 520 \mathrm{~nm}{ }^{[22]}$.

139 Angiogenesis analysis

140 The serum-free supernatants of tumor cells from different experimental groups were collected

141 and suspended the HUVEC cells to $2 \times 10^{4}$ cells $/ 100 \mathrm{uL}$. After being cultivated at $37^{\circ} \mathrm{C}$ for $4-6 \mathrm{~h}$,

142 the angiogenesis assay was performed by the Celigo instrument.

143 Microarray processing and data analysis

144 The samples were hybridized with the GeneChip microarrays (901838, Affymetrix) to determine

145 gene expression abundance according to the manufacturer's instructions. The expression profile

146 was preprocessed by the Limma package in Bioconductor.

147 A robust multiarray averaging algorithm was used to perform background correction, quantile

148 normalization, and probe summarization on the microarray data to obtain a gene expression

149 matrix. The cut-off for the background correction was $20 \%$, and the coefficient of variation was

$15025 \%$. The Benjamini-Hochberg method was used to correct the significant difference level

151 (FDR). The screening criteria for significantly different genes were: $\mid$ Fold Change $\mid>1.5$ and

152 FDR $<0.05^{[23]}$. The biological pathways analysis of genes was performed by Ingenuity Pathway

153 Analysis (IPA).

Peer] reviewing PDF | (2021:02:58541:3:0:NEW 18 Dec 2021) 
154

155

156

157

158

159

160

161

162

163

164

165

166

167

168

169

170

171

172

173

174

175

176

177

178

179

180

181

182

183

184

185

186

187

188

189

190

191

192

193

\section{RNA extraction and qRT-PCR analysis}

According to the manufacturer's protocol, the Trizol reagent (Invitrogen) was used to extract total RNA from frozen cells. For cDNA synthesis, $1 \mu \mathrm{g}$ of total RNA was used to synthase the cDNA by the Go Script reverse transcription system (Promega, Maddison, MA, U.S.A.). The genes were detected by the SYBR Master Mixture (DRR041B, Takara) using the LightCycler480 Real-Time PCR system (Roche). For qRT-PCR, the GAPDH gene was used as endogenous control. The primers sequences and the length of the amplifications were shown in Table S1. The $2^{-\Delta \Delta \mathrm{Ct}}$ method was used to calculate the fold change for gene expression relative to the control.

\section{Protein extraction and Western-blot analysis}

Total protein was isolated from cells using protein cell lysis buffer and extracted by centrifugation at $13000 \mathrm{rpm}$ for $20 \mathrm{~min}$ at $4^{\circ} \mathrm{C}$. The equal amount of whole cell lysate was separated by SDS-PAGE gel electrophoresis. After the proteins were transferred to the PVDF membranes (Bio-Rad, CA, U.S.A.), the membranes were blocked by $5 \%$ skimmed milk and immunoblotted with the primary antibodies at $4^{\circ} \mathrm{C}$. Then the membranes were blotted with the secondary antibodies at room temperature for $1 \mathrm{~h}$. The following primary antibodies were used: anti-DIDO (1:1000, HPA049904, Sigma), anti-CCND1 (1:500, Cat2978, CST), anti-CDK6 (1:500, Cat3136, CST), anti-GAPDH (1:2000, Sc-32233, Santa Curz). The secondary antibodies were anti-rabbit or anti-mouse IgG conjugated to horseradish peroxidase (Santa Cruz Biotechnology). The Dyne ECL STAR Western Blot Detection kit (Dyne Bio, Seoul, Korea) and a chemiluminescent image system (Fusion Solo system, Villber Lourmat) were used to analyze the protein abundance.

\section{Statistical analysis}

The data were shown as the mean \pm S.D. from 3 independent replicates. The student's t-test was performed to analyze the quantitative data. $\mathrm{P}<0.05$ was considered statistically significant.

\section{Results}

\section{GeneChip microarrays analysis of HUVEC and K562-HUVEC co-cultured cell lines}

In this study, the human umbilical vein endothelial cells (HUVEC) were used as endothelial cells models in vitro. When the HUVEC cells were co-cultured with the K562 leukemia cell lines for 4 days, the proliferation was inhibited significantly (Figure S1). Then we analyzed the gene expression changes in HUVEC cells when co-cultured with the K562 leukemia cell lines by GeneChip, to investigate the genes which will inhibit the endothelial cells' proliferation in leukemia progression.

Compared with HUVEC lines, 398 probes up-regulated expression and 323 probes downregulated expression in K562 co-cultured HUVEC lines (Figure S2A). Ingenuity Pathway Analysis (IPA) found that the extracellular signal-regulated kinase 5 (ERK5) signaling was significantly inhibited (Z-score $=-2.111$ ) (Figure S2B). On the other hand, the DEGs were mainly enriched in microtubule dynamics $(Z$-score $=2.783)$, migration of brain cancer cell lines 
$194 \quad(Z$-score $=2.549)$, liver tumor $(Z$-score $=-2.782)$ and cell death of mononuclear leukocytes $(Z$ -

195

196

197

198

199

200

201

202

203

204

205

206

207

208

209

210

211

212

213

214

215

216

217

218

219

220

221

222

223

224

225

226

227

228

229

230

231

232

233

score $=-2.561)($ Figure S2C).

\section{Construction of RNAi cell lines and cell proliferation analysis}

We selected the first 30 down-regulated expression genes ( $\log 2$ (change fold) $>1, \mathrm{P}<0.05$ ) for further analysis (Table S2). RNAi lentiviral vectors for these 30 genes were constructed and transfected into HUVEC cells. 22 transgenic cell lines were successfully obtained, including the negative control (NC) and positive control (PC). The cell count results showed that the proliferation of cells was normal in the $\mathrm{NC}$ group, and which was significantly inhibited in the PC group. The proliferation folds on the fifth day were 12.09 and 2.12 times higher than those on the first day in the NC and PC groups, respectively. The fold change (FC) of cell count ([FC in $\mathrm{NC}$ group on the 5th day compared to which on the 1st day] / [FC in experiment group on the 5th day compared to which on the 1st day]) was used to evaluate the influence of gene RNAi in cell proliferation. The proliferation of $s h D I D O, \operatorname{shZC3H18}$, and $S h S M U R F 2$ cell lines was significantly inhibited, and the change fold was 3.37, 2.54, 2.07, respectively (Figure S3).

\section{Patients with lower transcript abundance of DIDO showed a better overall survival}

To determine the effect of the DIDO, ZC 3H18, and SMURF2 in Leukemia patients, we analyzed the survival based on their expression status from the public data (http://gepia2.cancer-pku.cn/). As shown in Figure 1A, the acute myeloid leukemia (AML) patients with the lower DIDO expression level, showed a better overall survival $(\mathrm{HR}=1.9 ; \mathrm{P}=0.025)$. However, the different expressions of $Z C 3 H 18$, and $S M U R F 2$ did not affect the overall survival in AML patients (Figure 1B and C).

\section{The proliferation of $\operatorname{shDIDO}$ cell line is inhibited and the apoptosis is increased}

To further investigate the function of the DIDO (Death inducer obliterator) gene in endothelial cells, we analyzed the proliferation and apoptosis of shDIDO cells. Firstly, the expression of $D I D O$ in shDIDO cells was analyzed. qRT-PCR found that the expression level of the DIDO gene at the mRNA level was suppressed in shDIDO cell lines $(\mathrm{P}<0.05)$, and the reduction efficiency reached $95.1 \%$ (Figure 2A). Western-blot detection found that the expression of DIDO protein in the shDIDO cells decreased by four times, compared with the shCtrl group (Figure 2B).

The proliferation rate of shDIDO cell line was analyzed by Celigo (Figure $2 \mathrm{C}$ and $\mathrm{D}$ ) and MTT (Figure 2E), and the proliferation rate of the $\operatorname{sh} D I D O$ cells was significantly decreased. This may indicate that the $D I D O$ gene is significantly related to the proliferation ability of HUVEC cells. The number of cells in the apoptotic state was detected by Annexin V-APC single staining method, and it was found that apoptosis cells in shDIDO group increased significantly than the HUVEC cells $(\mathrm{P}<0.05)$ after 5 days (Figure $2 \mathrm{G})$. Additionally, by detecting the activity of 
234 caspase, it was found that the activity of caspase $3 / 7$ in the $\operatorname{sh} D I D O$ group was significantly 235 increased. These results indicate that the $D I D O$ gene was significantly related to the apoptosis of 236 HUVEC cells (Figure 2H).

237 Due to the importance of angiogenesis in tumor progression, we analyzed the effect of DIDO 238 gene depletion on angiogenesis. The ability of $\operatorname{sh} D I D O$ cells to form lumens was analyzed to 239 investigate the metastasis ability of tumors. It was found that the area of angiogenesis-related 240 blood vessels in the shDIDO group were $20 \%$ less than that in the shCtrl group $(\mathrm{P}<0.05)$

241 (Figure 2F), which indicates that the DIDO gene may not associated with HUVEC cells 242 angiogenesis.

243

\section{GeneChip analysis of shDIDO and shCtrl cell lines}

To investigate the biological pathways $D I D O$ involved, the GeneChip expression profiles of shCtrl and shDIDO cell lines were analyzed. It was found that 521 genes in the shDIDO cells were up-regulated and 1006 genes were down-regulated (Fold Change $>1.5$ and FDR $<0.05$ ), compared with shCtrl cells (Figure 3A, Table S3). IPA analysis found that the ERK/MAPK signaling was significantly inhibited $(Z$-score $=-2.041)$ (Figure 3B). Additionally, the functions including morbidity or mortality $(Z$-score $=6.734)$ and organismal death $(Z$-score $=6.709)$, were significantly activated. The functions including cell viability $(Z$-score $=-5.369)$, cell survival $(Z$ score $=-5.349$ ) were significantly suppressed (Figure 3C and Table S4).

The genes down-regulated in shDIDO cells, and which are involved in tumorigenesis and development, were selected for further analysis (Table S5). Among them, 30 probes were identified by qRT-PCR as their expression pattern were similar to the GeneChip (Table S6). DIDO is a part of the centrosome protein and plays an important role in spindle assembly, so we infer that the $D I D O$ gene may correlate with the cell cycle. We further analyzed the cell cycle regulation genes of Cyclin Dependent Kinase 6 (CDKO) and Cyclin D1 (CCND1). The transcription of CCND1 and CDK6 in the shDIDO cells were 0.364 and 0.404 times of the shCtrl group, respectively. Meanwhile, the western-blot analysis found that the protein expression levels of CCND1 and CDK6 in shDIDO cells were reduced by $81.3 \%$ and $58.1 \%$, respectively. However, the DIDO maybe not interact with CDK6 or CCND1 directly, as shown in Figure 3D. We searched the protein interaction database and did not find the direct interaction of DIDO with $C D K 6$ or $C C N D 1$ either. The genes that may directly interact with $D I D O$ were $S R S F 1, S R P K 2$, $E E D$, and $W W P 2$ in the interaction network of DIDO analyzed by IPA.

\section{Discussion}

Endothelial cells provide a fertile niche that allows for the propagation of primitive and aggressive leukemic clones. This study aimed to identify the genes involved in the interaction between endothelial cells and leukemia.

Firstly, the GeneChip assay showed that the expression of 323 probes was down-regulated in K562-HUVEC co-cultured cells (Figure S3A), and the ERK5 signaling was significantly 
274 inhibited. It has been reported that the ERK5 pathway mediates cell survival, apoptosis, and 275 proliferation signaling in embryonic stem cells ${ }^{[24]}$. We infer that the decreased ERK5 signaling 276 may correlate with the proliferation inhibition of HUVEC cells. There is increasing evidence to 277 indicate that the ERK5 signaling takes part in the development and progression of several types 278 of cancers, including breast cancer, myeloma, lymphoma, leukemia ${ }^{[25]}$. In addition, some studies 279 suggested that the ERK5 could represent a promising target for therapeutic intervention in 280 leukemia ${ }^{[26]}$.

281 To investigate the key genes involved in the inhibition of HUVEC cells when co-cultured with $282 \mathrm{~K} 562$, RNAi cell lines of the top 30 down-regulated expression genes were constructed and we 283 analyzed the proliferation of them. The proliferation of $s h D I D O$, shZC $3 H 18$, and $s h S M U R F 2$ 284 cells was significantly inhibited, and their transcripts were significantly inhibited in HUVEC285 K562 co-cultured cells. These may indicate that the down-regulate expression of these genes 286 would help inhibit the activity of ECs in the leukemia environment. To understand the role of 287 these genes in vivo, we analyzed the survival of leukemia patients when stratified by the 288 expression abundance. It was found that leukemia patients with lower expression of DIDO 289 showed better survival. Based on these findings, we focused this study on the DIDO gene. 290 DIDO plays an important role in mitotic progression and chromosome instability as it is a 291 component of the centrosome proteins and plays an essential role in spindle assembly ${ }^{[16]}$. It has 292 been reported that $D I D O$ is related to chromosomal instability. DIDO gene may be a novel MSI 293 biomarker, as its mutation has a high concordance level with MSI-H status (microsatellite 294 instability high), based on research enrolled 1301 colorectal cancer FFPE (formalin-fixed, 295 paraffin-embedded) tissue sections [27]. There are increasing evidence showing that the DIDO 296 plays an important role in tumor onset and progression. In bladder cancer, the reduction of DIDO 297 mRNA resulted in increased apoptosis, reduced proliferation in vitro, and inhibited 298 tumorigenesis in vivo. The authors pointed that the potential mechanism of DIDO action might 299 involve SAPK/JNK signaling cascades ${ }^{[15]}$. In addition, in melanoma cells, DIDO was found to 300 induce the expression of integrin $\alpha$, and promoting the attachment, migration, invasion and 301 apoptosis resistance of melanoma cells ${ }^{[17]}$. In this study, the transcript and protein abundance of 302 DIDO gene was inhibited in siRNA cell lines, which resulted in an inhibition of proliferation, 303 and an up regulation of apoptosis. This is consistent with the bladder cancer and melanoma. 304 To investigate the genes that are affected by DIDO, the DEGs between shDIDO and shCtrl cell 305 lines were analyzed. The ERK/MAPK signaling was significantly inhibited in both the HUVEC306 K562 co-cultured cells and shDIDO cells, the ERK5 pathway mediates apoptosis and 307 proliferation signaling in several kinds of tumor cells ${ }^{[25]}$. It was reported that the ERK5 was 308 regulated by phosphorylation and established a link between the CDK pathway during mitosis $309{ }^{[28]}$. On the other hand, the cyclin Dl gene is a key step in cell proliferation, and it may be a novel 310 target of the ERK5 cascade ${ }^{[29]}$. In this study, we also found that the expression of cell cycle 311 genes, $C D K 6$ and $C C N D 1$, were down-regulated in $s h D I D O$, which was verified by qRT-PCR 312 and western-blot. According to these results, there may be crosstalk between DIDO, ERK5, 
$313 C D K 6$, and $C C N D 1$, and these genes may work together to inhibit cell proliferation. However, 314 how these genes interact with each other still need further study.

315 As $D I D O$ may act as a transcription factor ${ }^{[30]}$, we screened for potential $D I D O$ target genes that 316 down regulated in $S h D I D O$ cells. The IPA interaction network indicated that DIDO directly 317 interact with $W W P 2, S R P K 2$ and $S R S F 1$. WWP2 (WW domain containing E3 ubiquitin protein

318 ligase 2) gene encodes a protein that play a role in the regulation of oncogene signaling pathways 319 via interactions with SMAD proteins and the tumor suppressor PTEN. WWP2 could promote the

320

321

322

323

324

325

326

327

328

329

330

331

332

333

334

335

336

337

338

339

340

341

342

343

344

345

346

347

348

349

350

351 proliferation of gastric cancer cells in a PTEN-dependent manner, and its silencing will inhibit proliferation and growth of gastric cancer cells [31], suggesting a vital role of WWP2 in cancer progression. SRPK2 (Serine/Arginine-Rich Protein-Specific Kinase-2, SRSF protein kinase-2) is up-regulated in multiple human tumors, and plays an important role in the progression and metastasis of prostate cancer [32]. SRSF1 (serine/arginine-rich splicing factor 1) promotes proliferation and injury-induced neointima formation in vascular smooth muscle cells ${ }^{[33]}$, and it could promote tumorigenesis through regulation of alternative splicing in colon cancer [34], glioblastoma ${ }^{[35]}$, and other cancers. The overexpression of $S R S F 1$ could promote cell proliferation and delay cell apoptosis during acinar morphogenesis in breast cancer ${ }^{[36]}$. According to these previously researches, DIDO may play roles by interaction with $W W P 2$, SRPK2 and SRSF1.

In order to analyze the genes or epigenetic modification that may regulate the expression of $D I D O$, the published ChIP-seq data in different leukemia cell lines (including K562) was analyzed. There are multiple DNase-seq peaks near $D I D O$, presuming that there are regulatory factors binding at the corresponding position. As shown in Figure 4 and Figure sS4, the $C D K 7$, $C D K 8, A T F 1, B C L A F 1$, and $C B X 3$ had binding peaks at the transcription start site (TSS) near exon 1 and exon 2. H3K4me3 and Pol II (POLR2A) bind to the site near exon1 and exon 2 of DIDO gene. The EGR1, FOS, MAX, NCOR1, H3K4me1, H3K27ac, MED1, and EP300 had binding peaks specifically at the TSS on exon1, and CHD7, SIRT6, c-MYC indicate binding signals near the exon 2 TSS. This indicate that the transcript of DIDO gene was regulated by the transcript factors and epigenetic factors. However, the correlation between these factors and the $D I D O$ gene needs further experimental verification.

\section{Conclusions}

In conclusion, the apoptosis and proliferation mechanism in leukemia endothelial cells that may regulate by $D I D O$ was summarized in figure 5 . The ERK5 signal will be inhibited by the downregulation of DIDO in $S h D I D O$ cell lines, and the genes in ERK5 signaling may play roles in cell apoptosis and proliferation, and regulate the gene transcription and translation. In addition, the inhibited DIDO in HUVEC will indirectly inhibit the expression of CDK6 and CCND1, which will inhibit the proliferation of cells. The proliferation inhibition of endothelial cells may inhibit the development of leukemia, and inducing cell apoptosis may become a therapy to treat leukemia. The $D I D O$ gene discovered in this study provides a theoretical basis for the 
352 development of drug targets for leukemia. But it is also necessary to study the gene expression of 353 DIDO in leukemia patients.

354

355

356

357

358

359

360

361

362

363

364

365

366

367

368

369

370

371

372

373

374

375

376

377

378

379

380

381

382

383

384

385

386

387

388

389

390

391

392

393

\section{References}

[1] Zhang H, Vakil V, Braunstein M, Smith EL, Maroney J, Chen L, Dai K, Berenson JR, Hussain MM, Klueppelberg U, Norin AJ, Akman HO, Ozçelik T, Batuman OA. Circulating endothelial progenitor cells in multiple myeloma: implications and significance. Blood 2005; 105: 3286-3294, doi: 10.1182/blood-2004-06-2101

[2] Cortelezzi A, Fracchiolla NS, Mazzeo LM, Silvestris I, Pomati M, Somalvico F, Bertolini F, Mancuso P, Pruneri GC, Gianelli U, Pasquini MC, Cortiana M, Deliliers GL.Endothelial precursors and mature endothelial cells are increased in the peripheral blood of myelodysplastic syndromes. Leuk Lymphoma 2005; 46: 1345-1351, doi: 10.1080/10428190500144235

[3] Wierzbowska A, Robak T, Krawczyńska A, Wrzesień-Kuś A, Pluta A, Cebula B, Smolewski P. Circulating endothelial cells in patients with acute myeloid leukemia. Eur J Haematol. 2005;75(6):492-497. doi:10.1111/j.1600-0609.2005.00549.x

[4] Rigolin GM, Maffei R, Rizzotto L, Ciccone M, Sofritti O, Daghia G, Cibien F, Cavazzini F, Marasca R, Cuneo A. Circulating endothelial cells in patients with chronic lymphocytic leukemia: clinical-prognostic and biologic significance. Cancer 2010; 116: 1926-1937, doi: 10.1002/cncr.24961

[5] Gunsilius E, Duba HC, Petzer AL, Kähler CM, Grünewald K, Stockhammer G, Gabl C, Dirnhofer S, Clausen J, Gastl G. Evidence from a leukaemia model for maintenance of vascular endothelium by bone-marrow-derived endothelial cells. Lancet. 2000;355(9216):1688-1691. doi:10.1016/S0140-6736(00)02241-8

[6] Butler JM, Kobayashi H, Rafii S. Instructive role of the vascular niche in promoting tumour growth and tissue repair by angiocrine factors. Nat Rev Cancer. 2010;10(2):138-146. doi:10.1038/nrc2791

[7] Le Q, Hadland B, Meshinchi S, Bernstein I. Notch blockade overcomes endothelial cell-mediated resistance of FLT3/ITD-positive AML progenitors to AC220 treatment. Leukemia. 2021;35(2):601-605. doi:10.1038/s41375-020-0893-y

[8] Kaplan RN, Rafii S, Lyden D. Preparing the "soil": the premetastatic niche. Cancer Res. 2006;66(23):11089-11093. doi:10.1158/0008-5472.CAN-06-2407

[9] Colmone A, Amorim M, Pontier AL, Wang S, Jablonski E, Sipkins DA. Leukemic cells create bone marrow niches that disrupt the behavior of normal hematopoietic progenitor cells. Science. 2008;322(5909):1861-1865. doi:10.1126/science.1164390

[10] Hatfield KJ, Evensen L, Reikvam H, Lorens JB, Bruserud. Soluble mediators released by acute myeloid leukemia cells increase capillary-like networks. Eur J Haematol. 2012;89(6):478-490. doi:10.1111/ejh.12016

[11] Pezeshkian B, Donnelly C, Tamburo K, Geddes T, Madlambayan GJ. Leukemia Mediated Endothelial Cell Activation Modulates Leukemia Cell Susceptibility to Chemotherapy through a Positive Feedback Loop Mechanism. PLoS One. 2013;8(4):e60823. doi: 10.1371/journal.pone.0060823. 
394

395

396

397

398

399

400

401

402

403

404

405

406

407

408

409

410

411

412

413

414

415

416

417

418

419

420

421

422

423

424

425

426

427

428

429

430

431

432

433

434

435

[12] Bosse RC, Wasserstrom B, Meacham A, Wise E, Drusbosky L, Walter GA, Chaplin DJ, Siemann DW, Purich DL, Cogle CR. Chemosensitizing AML cells by targeting bone marrow endothelial cells. Exp Hematol. 2016;44(5):363-377.e5. doi:10.1016/j.exphem.2016.02.003

[13] Bobryshev YV, Orekhov AN, Chistiakov DA. Vascular stem/progenitor cells: current status of the problem. Cell Tissue Res. 2015;362(1):1-7. doi:10.1007/s00441-015-2231-7

[14] Cogle CR, Goldman DC, Madlambayan GJ, Leon RP, Masri AA, Clark HA, Asbaghi SA, Tyner JW, Dunlap J, Fan G, Kovacsovics T, Liu Q, Meacham A, Hamlin KL, Hromas RA, Scott EW, Fleming WH. Functional integration of acute myeloid leukemia into the vascular niche. Leukemia. 2014 Oct;28(10):1978-1987. doi: 10.1038/leu.2014.109.

[15] Li J, Wang AS, Wang S, Wang CY, Xue S, Li WY, Ma TT, Shan YX. Death-inducer obliterator 1 (DIDO1) silencing suppresses growth of bladder cancer cells through decreasing SAPK/JNK signaling cascades. Neoplasma. 2020;67(5):1074-1084. doi:10.4149/neo_2020_191115N01171

[16] Xiao J, Zhang R, Peng J, Yang Z. BAP1 maintains chromosome stability by stabilizing DIDO1 in renal cell carcinoma. Am J Cancer Res. 2020;10(5):1455-1466. Published 2020 May 1.PMID: 32509391

[17] Braig S, Bosserhoff AK. Death inducer-obliterator 1 (Dido1) is a BMP target gene and promotes BMP-induced melanoma progression. Oncogene. 2013;32(7):837-848. doi:10.1038/onc.2012.115

[18] Tang J, Diao P, Shu X, Li L, Xiong L. Quercetin and Quercitrin Attenuates the Inflammatory Response and Oxidative Stress in LPS-Induced RAW264.7 Cells: In Vitro Assessment and a Theoretical Model. Biomed Res Int. 2019;2019:7039802. Published 2019 Oct 28. doi:10.1155/2019/7039802

[19] Nabzdyk CS, Chun M, Pradhan L, Logerfo FW. High throughput RNAi assay optimization using adherent cell cytometry. J Transl Med. 2011;9:48. Published 2011 Apr 25. doi:10.1186/1479-58769-48

[20] Moodley S, Koorbanally NA, Moodley T, Ramjugernath D, Pillay M. The 3-(4,5-dimethylthiazol2-yl)-2,5-diphenyl tetrazolium bromide (MTT) assay is a rapid, cheap, screening test for the in vitro anti-tuberculous activity of chalcones. J Microbiol Methods. 2014;104:72-78. doi:10.1016/j.mimet.2014.06.014

[21] Pan Y, Shan W, Fang H, Guo M, Nie Z, Huang Y, Yao S. Sensitive and visible detection of apoptotic cells on Annexin-V modified substrate using aminophenylboronic acid modified gold nanoparticles (APBA-GNPs) labeling. Biosens Bioelectron. 2014;52:62-68. doi:10.1016/j.bios.2013.07.057

[22] Stennicke HR, Salvesen GS. Biochemical characteristics of caspases-3, -6, -7, and -8. J Biol Chem. 1997;272(41):25719-25723. doi:10.1074/jbc.272.41.25719

[23] Ritchie ME, Phipson B, Wu D, Hu Y, Law CW, Shi W, Smyth GK. Limma powers differential expression analyses for RNA-sequencing and microarray studies. Nucleic Acids Res. 2015;43(7):e47. doi:10.1093/nar/gkv007

[24] Williams CA, Fernandez-Alonso R, Wang J, Toth R, Gray NS, Findlay GM. ERK5 Is a Key Regulator of Naive-Primed Transition and Embryonic Stem Cell Identity. Cell Rep. 2016;16(7):1820-1828. doi:10.1016/j.celrep.2016.07.033

[25] Stecca B, Rovida E. Impact of ERK5 on the Hallmarks of Cancer. Int J Mol Sci. 2019 Mar 21;20(6):1426. doi: 10.3390/ijms20061426.

Peer) reviewing PDF | (2021:02:58541:3:0:NEW 18 Dec 2021) 
436

437

438

439

440

441

442

443

444

445

446

447

448

449

450

451

452

453

454

455

456

457

458

459

460

461

462

463

464

465

466

467

468

469

470

471

472

473

474

475

476

477
[26] Kang C, Kim JS, Kim CY, Kim EY, Chung HM. The Pharmacological Inhibition of ERK5 Enhances Apoptosis in Acute Myeloid Leukemia Cells. Int J Stem Cells. 2018;11(2):227-234. doi:10.15283/ijsc18053;

[27] Velasco A, Tokat F, Bonde J, Trim N, Bauer E, Meeney A, de Leng W, Chong G, Dalstein V, Kis LL, Lorentzen JA, Tomić S, Thwaites K, Putzová M, Birnbaum A, Qazi R, Primmer V, DockhornDworniczak B, Hernández-Losa J, Soares FA, Gertler AA, Kalman M, Wong C, Carraro DM, Sousa AC, Reis RM, Fox SB, Fassan M, Brevet M, Merkelbach-Bruse S, Colling R, Soilleux E, Teo RYW, D'Haene N, Nolet S, Ristimäki A, Väisänen T, Chapusot C, Soruri A, Unger T, Wecgowiec J, Biscuola M, Frattini M, Long A, Campregher PV, Matias-Guiu X. Multi-center realworld comparison of the fully automated Idylla ${ }^{\mathrm{TM}}$ microsatellite instability assay with routine molecular methods and immunohistochemistry on formalin-fixed paraffin-embedded tissue of colorectal cancer. Virchows Arch. 2021;478(5):851-863. doi:10.1007/s00428-020-02962-x

[28] Iñesta-Vaquera FA, Campbell DG, Tournier C, Gómez N, Lizcano JM, Cuenda A. Alternative ERK5 regulation by phosphorylation during the cell cycle. Cell Signal. 2010;22(12):1829-1837. doi:10.1016/j.cellsig.2010.07.010

[29] Mulloy R, Salinas S, Philips A, Hipskind RA. Activation of cyclin D1 expression by the ERK5 cascade. Oncogene. 2003;22(35):5387-5398. doi:10.1038/sj.onc.1206839

[30] Rojas AM, Sanchez-Pulido L, Fütterer A, van Wely KH, Martinez-A C, Valencia A. Death inducer obliterator protein 1 in the context of DNA regulation. Sequence analyses of distant homologues point to a novel functional role. FEBS J. 2005;272(14):3505-3511. doi:10.1111/j.17424658.2005.04759.x

[31] Wang K, Liu J, Zhao X, Li H, Luo G, Yu Y, Guo Y, Zhang L, Zhu J, Wang S, Hua W, Yang A, Zhang R, Li J. WWP2 regulates proliferation of gastric cancer cells in a PTEN-dependent manner. Biochem Biophys Res Commun. 2020;521(3):652-659. doi:10.1016/j.bbrc.2019.10.179

[32] Zhuo YJ, Liu ZZ, Wan S, Cai ZD, Xie JJ, Cai ZD, Song SD, Wan YP, Hua W, Zhong W, Wu CL. Enhanced expression of SRPK2 contributes to aggressive progression and metastasis in prostate cancer. Biomed Pharmacother. 2018;102:531-538. doi:10.1016/j.biopha.2018.03.079

[33] Xie N, Chen M, Dai R, Zhang Y, Zhao H, Song Z, Zhang L, Li Z, Feng Y, Gao H, Wang L, Zhang T, Xiao RP, Wu J, Cao CM. SRSF1 promotes vascular smooth muscle cell proliferation through a $\Delta$ 133p53/EGR1/KLF5 pathway. Nat Commun. 2017;8:16016. Published 2017 Aug 11. doi:10.1038/ncomms 16016

[34] Chen L, Luo C, Shen L, Liu Y, Wang Q, Zhang C, Guo R, Zhang Y, Xie Z, Wei N, Wu W, Han J, Feng Y. SRSF1 Prevents DNA Damage and Promotes Tumorigenesis through Regulation of DBF4B Pre-mRNA Splicing. Cell Rep. 2017;21(12):3406-3413. doi:10.1016/j.celrep.2017.11.091

[35] Zhou X, Wang R, Li X, Yu L, Hua D, Sun C, Shi C, Luo W, Rao C, Jiang Z, Feng Y, Wang Q, Yu S. Splicing factor SRSF1 promotes gliomagenesis via oncogenic splice-switching of MYO1B. J Clin Invest. 2019;129(2):676-693. doi:10.1172/JCI120279

[36] Anczuków O, Rosenberg AZ, Akerman M, Das S, Zhan L, Karni R, Muthuswamy SK, Krainer AR. The splicing factor SRSF1 regulates apoptosis and proliferation to promote mammary epithelial cell transformation. Nat Struct Mol Biol. 2012;19(2):220-228. Published 2012 Jan 15. doi:10.1038/nsmb.2207 
Figure 1

Figure 1 The KM-plot in Acute Myeloid Leukemia stratified by the expression level of DIDO (A), ZC3H18 (B), and SMURF2 (C).
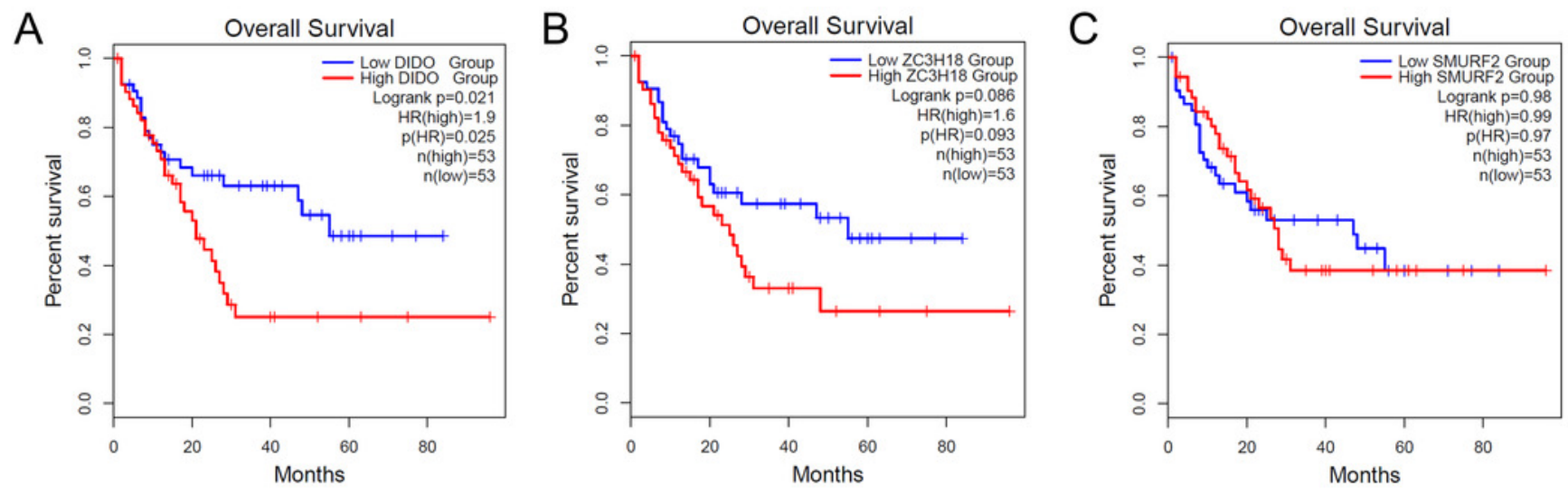
Figure 2

Figure 2. The function assays of DIDO gene.

A) The RNA transcript abundance of DIDO gene in shDIDO and shCtrl cells detected by qRTPCR; B) Western-blot assay for the expression of DIDO protein; C) Cell proliferation pictures of shDIDO and shCtrl cell lines by Celigo; D and E) Cell count of shDIDO and shCtrl cell lines; F) Analysis of the area of blood vessel formation; G) Cell apoptosis ratio analyzed by FACS; H) Caspase3/7 activity assays. 
A

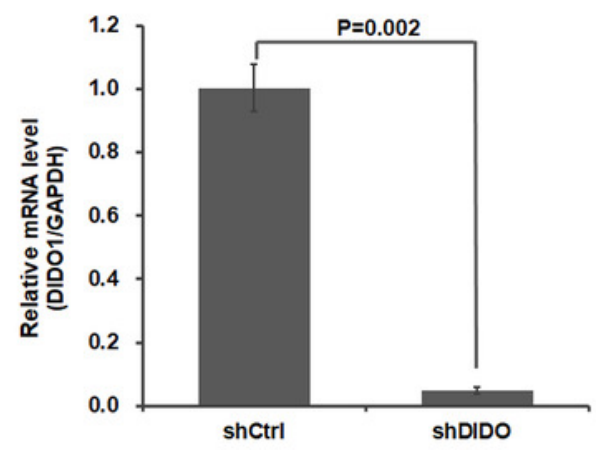

C

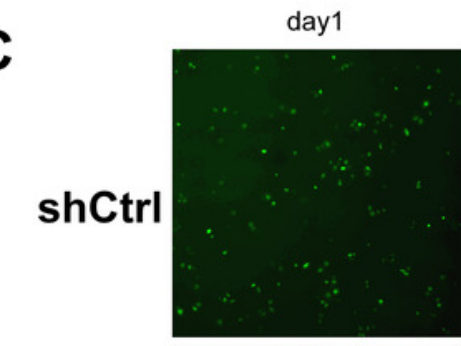

shDIDO

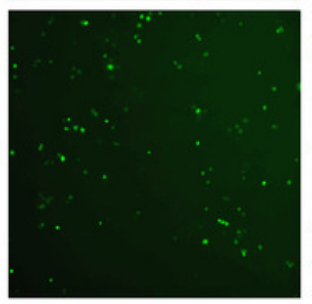

D

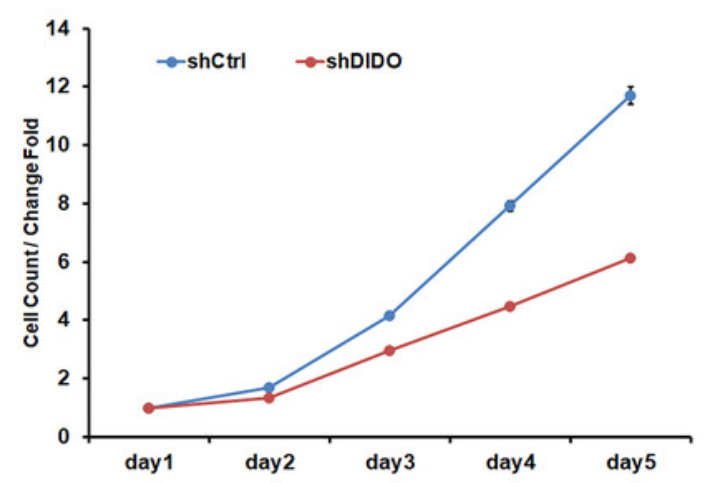

F
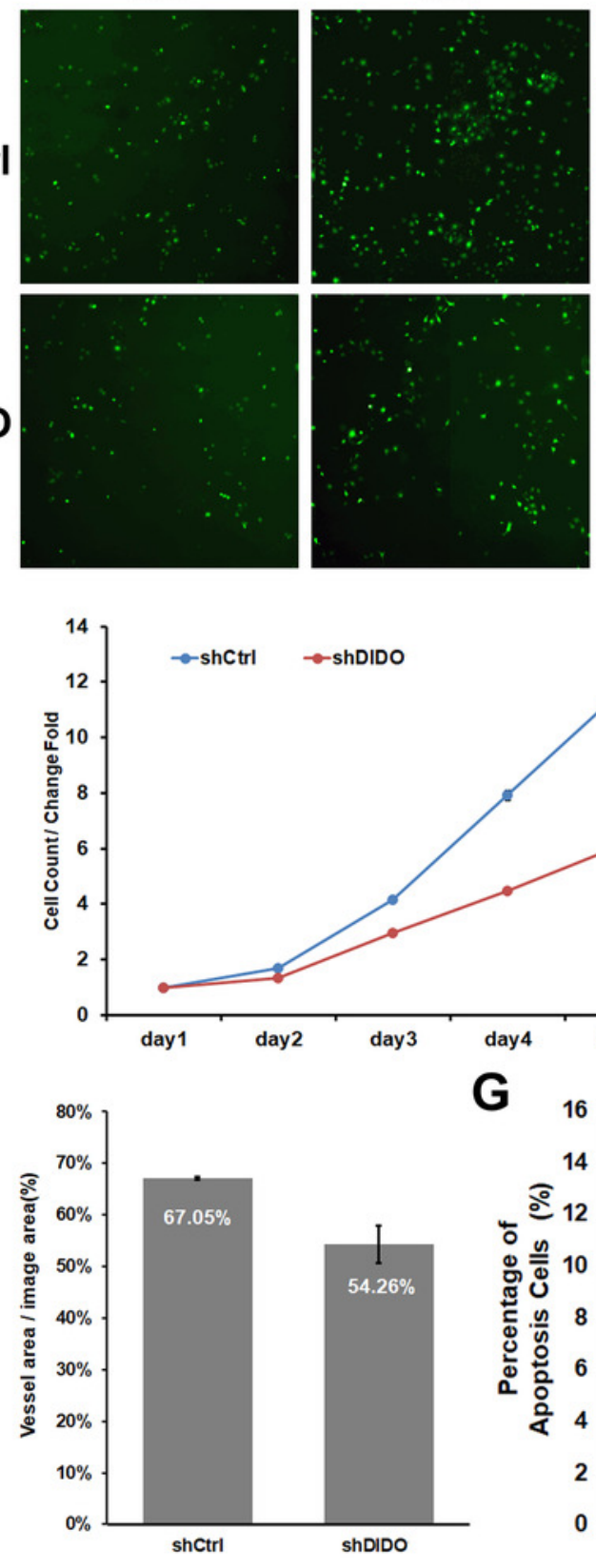

B

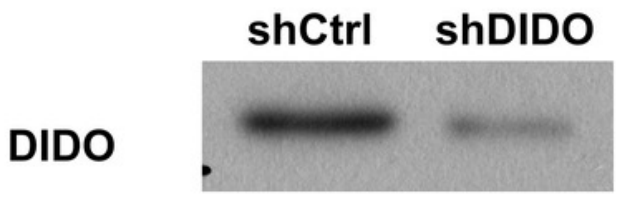

GAPDH

day3

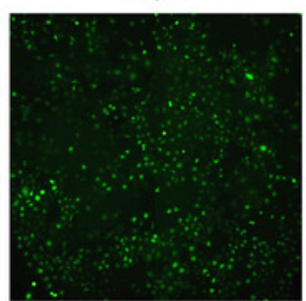

day4

day5
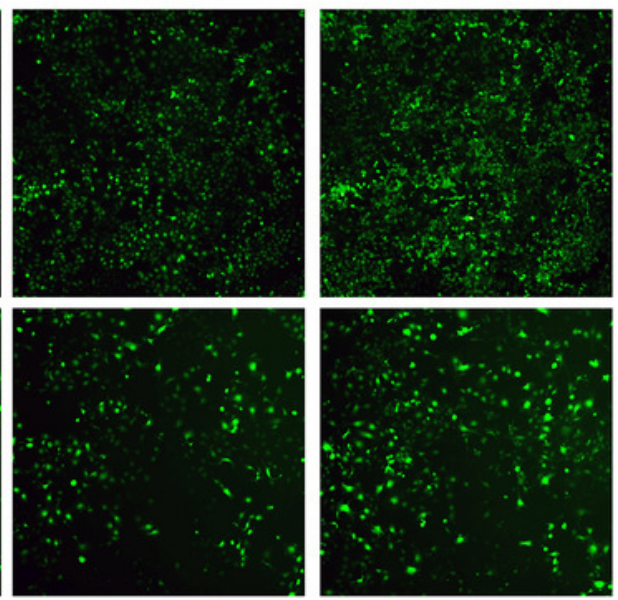

E

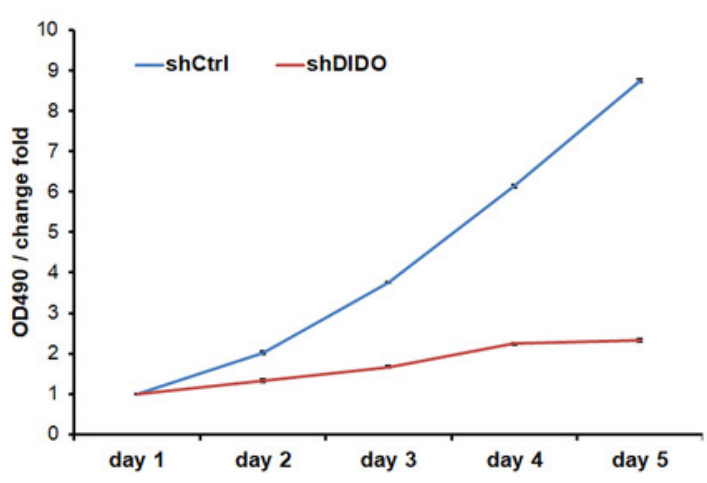

H

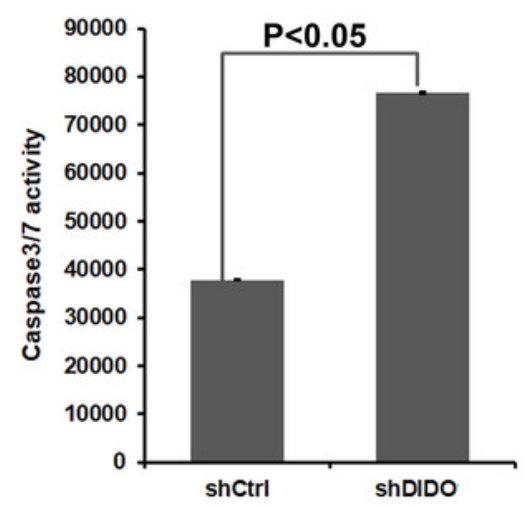




\section{Figure 3}

Figure 3 Analysis of downstream genes regulated by DIDO

A) The volcano map of the differently expressed probes in shCtrl and shDIDO cell lines; B) The enrichment of DEGs in the classical signal pathway; C) Disease and function heat maps show the expression changes of DEGs indifferent diseases and functions. Orange means the disease or functional state is activated (Z-score $>0$ ), blue means the disease or functional state is inhibited $(Z$-score $<0)$, and gray means the disease or functional state is not determined (Z-score cannot be calculated); The disease or function is significantly activated if Z-score $>2$ ), and significantly inhibited if Z-score $<-2$. Significantly activated diseases or functions include: morbidity or mortality (Z-score=6.734), organic death (6.709), etc.; Significantly inhibited diseases or functions include: cell viability (-5.369), cell survival (-5.349); D) Gene interaction network diagram shows the interaction network between molecules; E) The expression of CCND1 and CDK6 analyzed by qRT-PCR and Western-blot. 

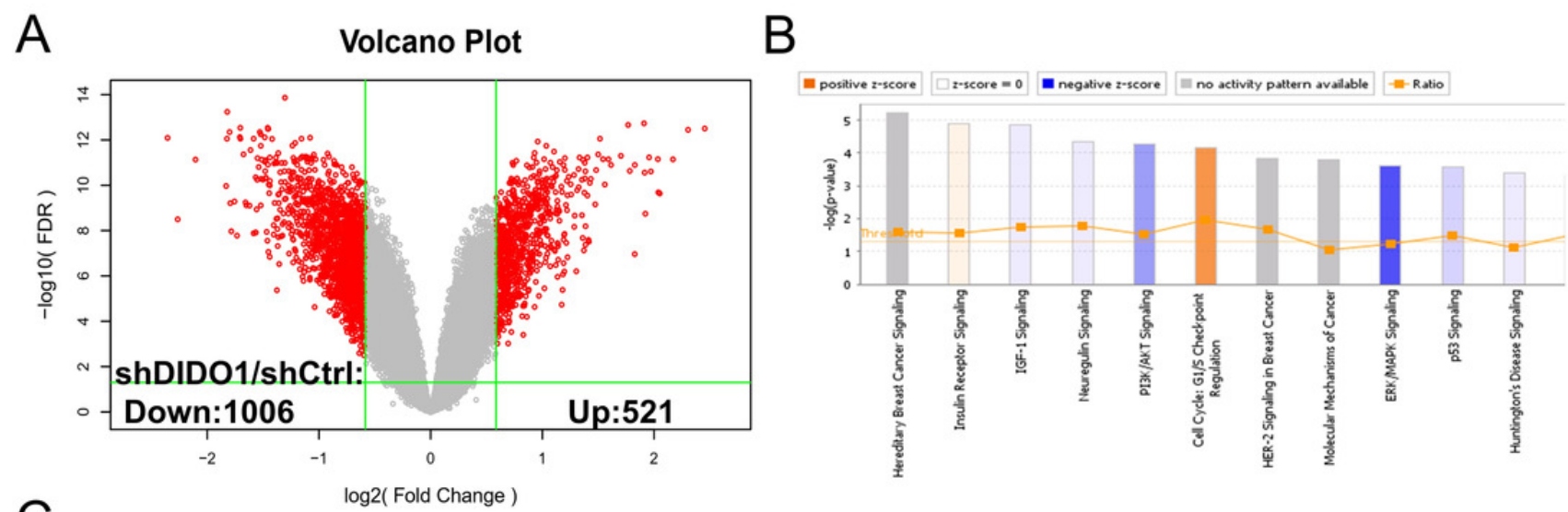

C
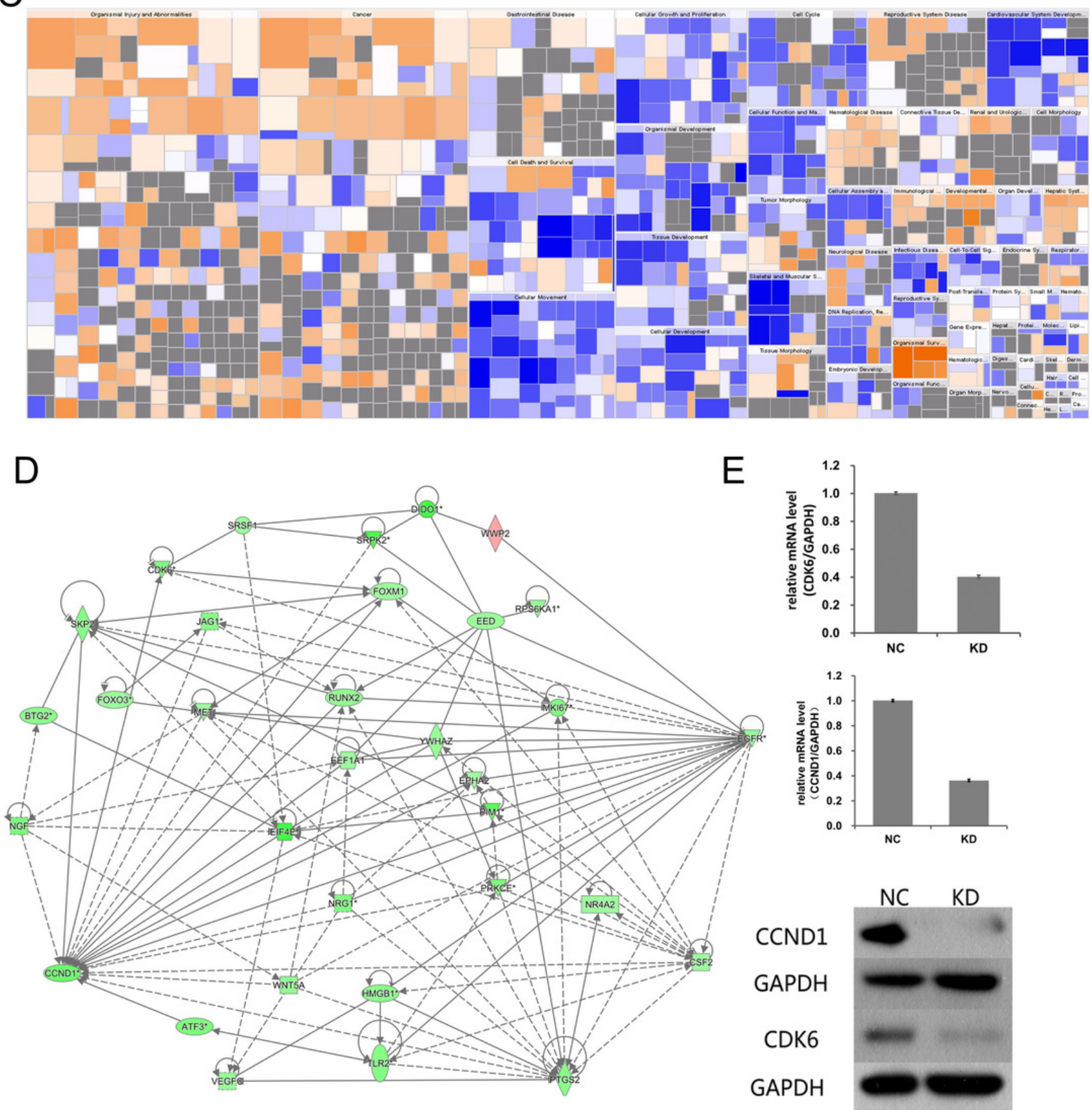
Figure 4

Figure 4 The transcript factors and epigenetic factors bind with DIDO gene analyzed by public data.

Scale

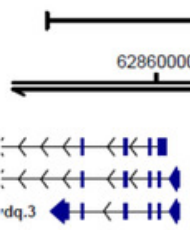

$30 \mathrm{~kb}$

$62870000 \quad 62880000$

$62940000 \quad 62950000 \quad 62960000$

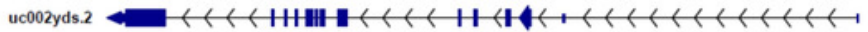

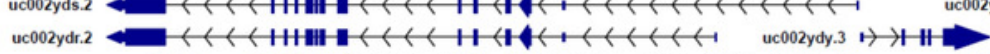

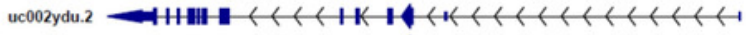

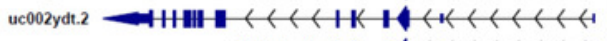

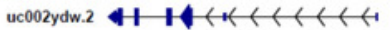

uc002ydx.2

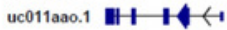

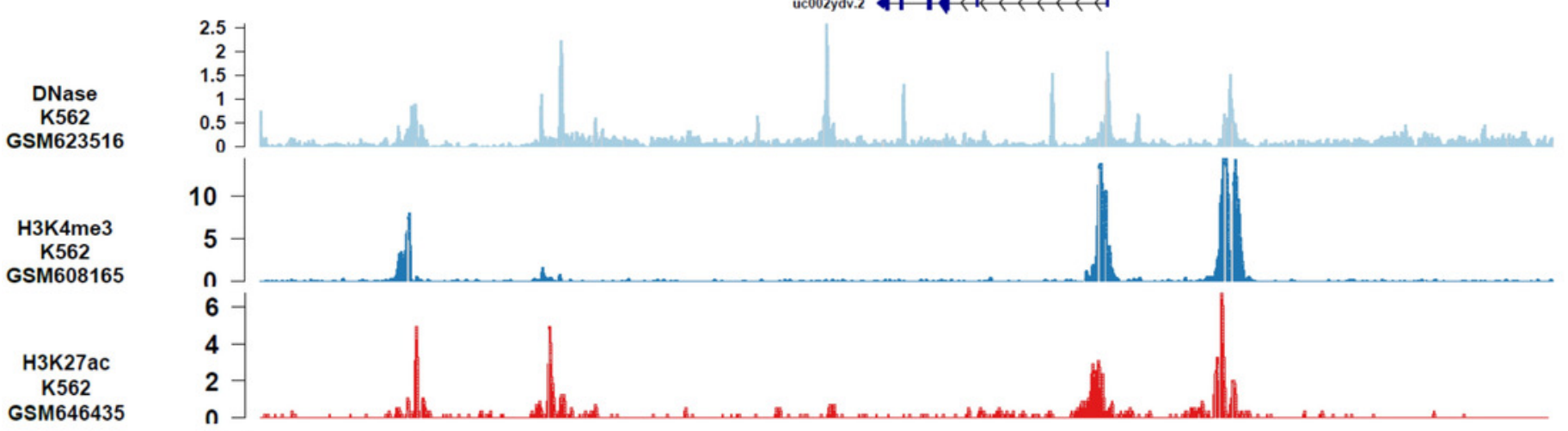

GSM646435

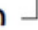

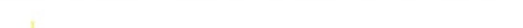

$1-1$
$0.8-$
$0.6-$
$0.4-$
$0.2-$

K562

GSM935340

BCLAF1

K562

GSM803515

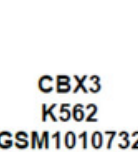

0.8
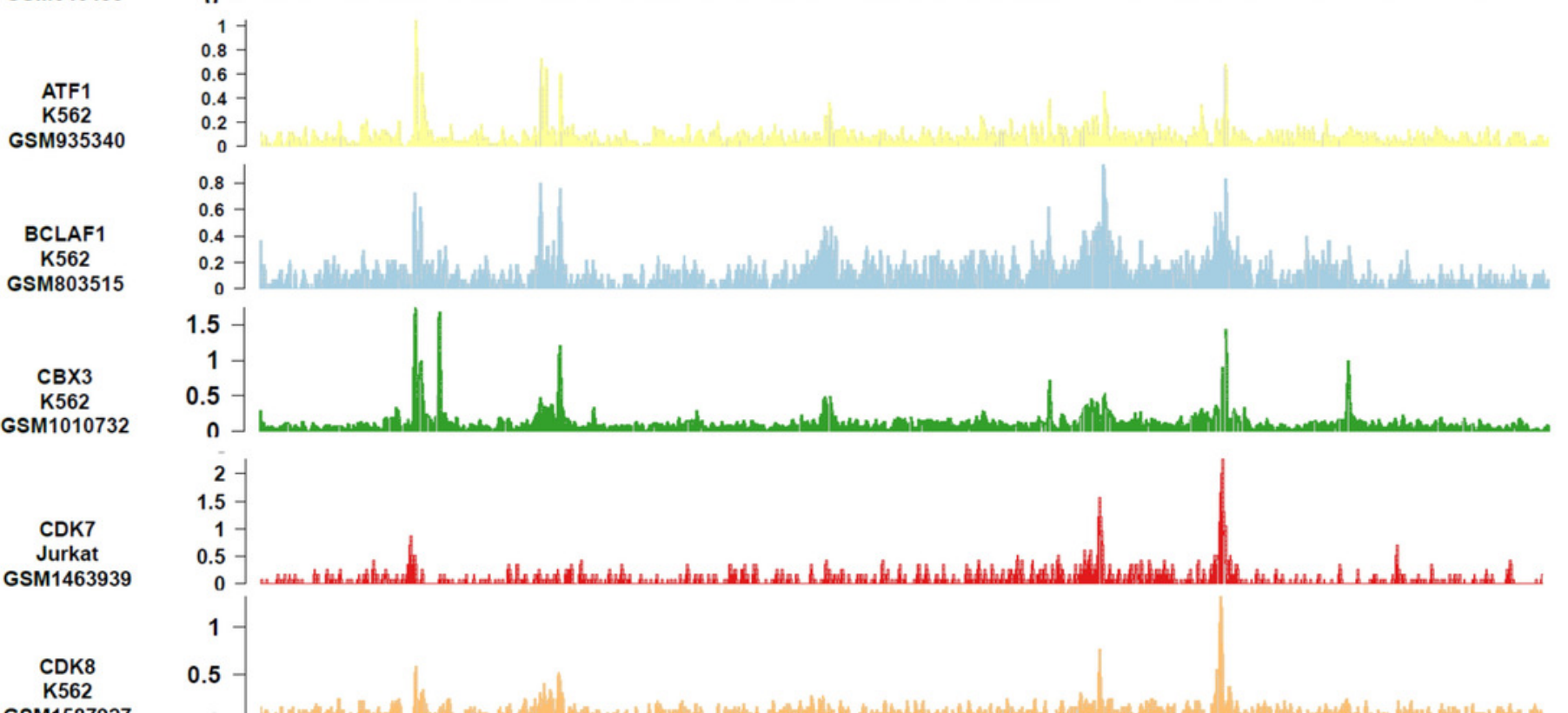

$$
\begin{array}{r}
2-1 \\
1.5-1
\end{array}
$$
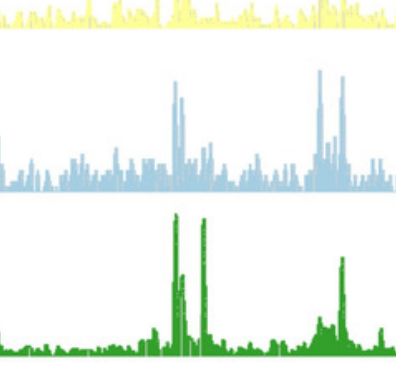

$\begin{array}{r}1 \\ 0.5 \\ 0\end{array}-$
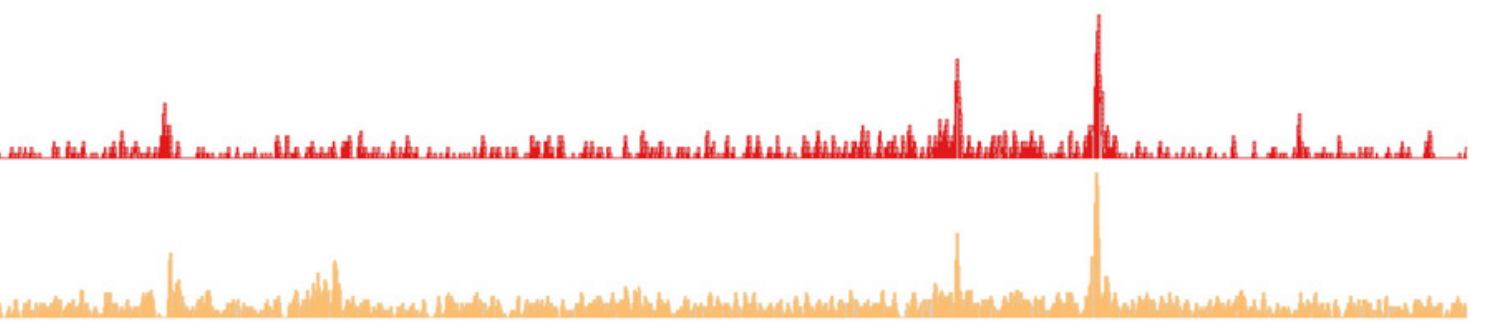

GSM1587927

$$
\left.\begin{array}{r}
1- \\
0.5- \\
0
\end{array}\right]
$$




\section{Figure 5}

Figure 5 The apoptosis and proliferation mechanism in leukemia endothelial cells that may regulated by DIDO.

Green represents down-regulated expression of genes in shDIDO cells, and pink represents up-regulated expression of genes in ShDIDO cells. The solid line indicates clarified relationship between genes; The dotted line indicates the relationship between genes needs further study. 


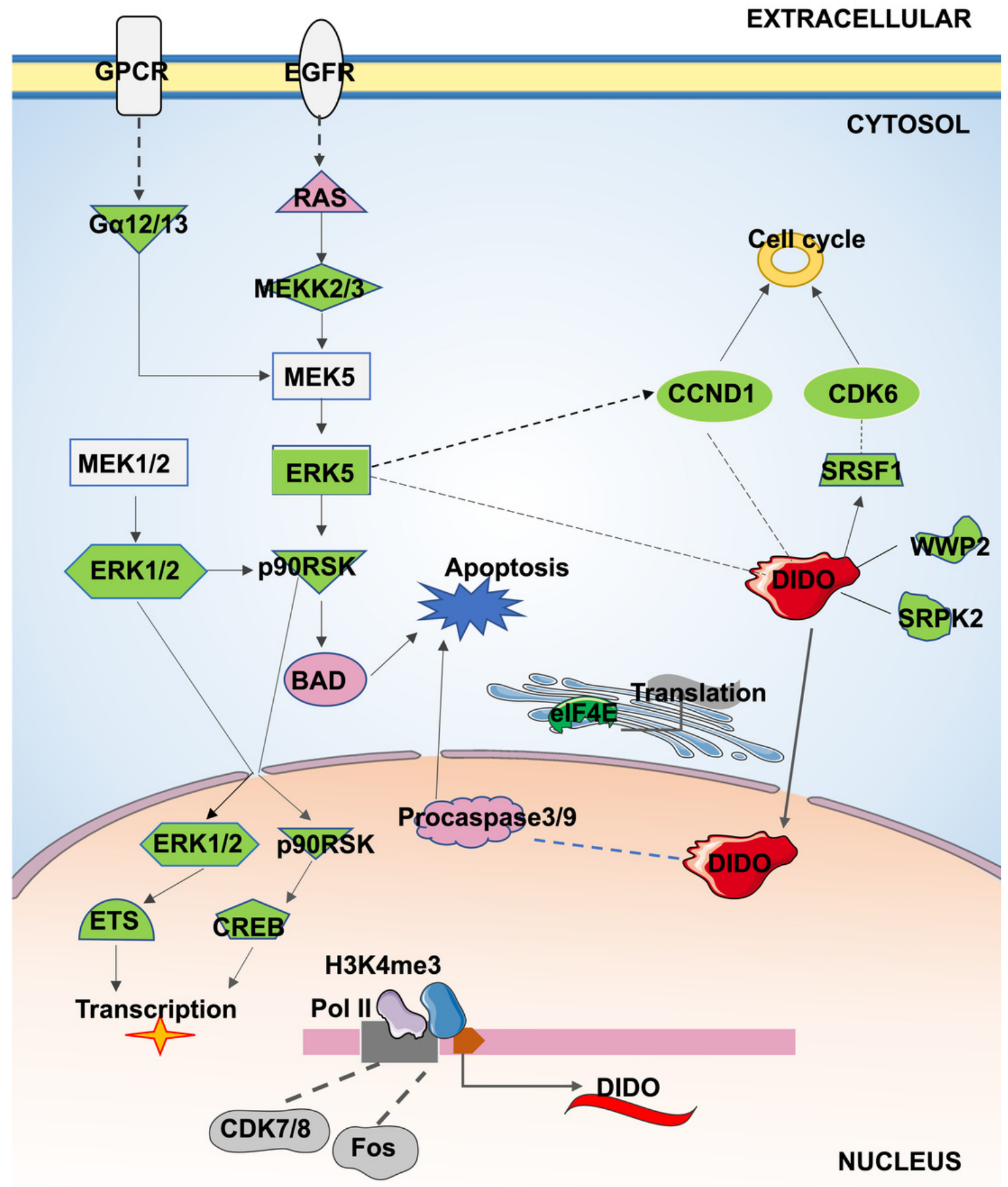

